\title{
Effect of impedance and higher order chromaticity on the measurement of linear chromaticity
}

\author{
V.H. Ranjbar \\ Tech-X, Boulder, Colorado 80303, USA \\ C. Y. Tan
}

Fermi National Accelerator Laboratory, Batavia, Illinois 60510, USA

(Received 22 July 2010; revised manuscript received 14 July 2011; published 29 August 2011)

\begin{abstract}
The combined effect of impedance and higher order chromaticity can act on the beam in a nontrivial manner which can cause a tune shift which depends on the relative momenta with respect to the "on momentum" particle $(\Delta p / p)$. Experimentally, this tune shift affects the measurement of the linear chromaticity which is traditionally measured with a change of $\Delta p / p$. The theory behind this effect will be derived in this paper. Computer simulations and experimental data from the Tevatron will be used to support the theory.
\end{abstract}

DOI: 10.1103/PhysRevSTAB.14.082802

PACS numbers: 29.27.Bd, 29.27.Fh

\section{INTRODUCTION}

The control of chromaticity in modern high energy colliders such as the Large Hadron Collider, Tevatron, and Relativistic Heavy Ion Collider is of critical importance for maintaining beam stability and in maximizing the beam lifetime both during acceleration and at high energy physics.

In order to deliver as many collisions as possible to the experiments, losses have to be reduced as much as possible. One significant source of continuous particle loss is related to the head-tail instabilities driven by wakefields. These instabilities can be controlled by increasing the betatron tune spread, and thus Landau damping, with large chromaticities. However, when the chromaticity is too large, the beam's betatron tune footprint can cover more resonances and thus decrease its lifetime. Clearly an understanding of the true chromaticity of the machine is critical for optimizing the integrated luminosity delivered to the experiments.

One major motivation for this paper comes from the observation that the linear chromaticity measured using the "traditional" method for uncoalesced [1] and coalesced [2] proton beam yields consistently different results by $\sim 1$ unit especially in the vertical plane, i.e., the measured linear chromaticity has a dependence on bunch structure. (The traditional method referred to here is the method where the rf frequency is varied, and thus the relative momenta with respect to the "on momentum" particle $\Delta p / p$, and the linear chromaticity is measured from the betatron tune excursions.)

Published by the American Physical Society under the terms of the Creative Commons Attribution 3.0 License. Further distribution of this work must maintain attribution to the author(s) and the published article's title, journal citation, and DOI.
In this paper we will show that the combined effect of higher order chromaticity and resistive wall impedance will cause a betatron tune shift which depends on $\Delta p / p$. This means that the linear chromaticity when measured with the traditional method will yield an incorrect result because the betatron tune does not shift as much as expected for coalesced protons.

\section{THE COMBINED EFFECT OF CHROMATICITY AND IMPEDANCE ON THE COLLECTIVE FREQUENCY}

In the simplest case a single particle's transverse motion can be characterized by

$$
\frac{d^{2} Y(t)}{d t^{2}}+\left(Q \omega_{0}\right)^{2} Y(t)=0
$$

Here $Y$ is the transverse position of the particle and $t$ is the time coordinate and $Q \omega_{0}$ is the angular betatron frequency where $\omega_{0}$ is the angular revolution frequency and $Q$ is the betatron tune. The solution of this differential equation will give us the transverse harmonic motion of a particle oscillating at the betatron angular frequency $\omega_{\beta}=Q \omega_{0}$.

If we now add in the effect of wakefields to the transverse motion, Eq. (1) becomes the forced simple harmonic oscillator equation [3]:

$$
\frac{d^{2} Y(t)}{d t^{2}}+\left(Q \omega_{0}\right)^{2} Y(t)=\frac{F_{\perp}(t)}{\gamma m} .
$$

For a broadband impedance, the transverse force $F_{\perp}$ is

$$
F_{\perp}(t)=-j \frac{\beta q I Z_{\perp}}{2 \pi R}\langle y\rangle,
$$

where $R$ is the mean radius of the accelerator, $q$ is the electronic charge, and $\beta$ is the relative speed of the particle with respect to the speed of light $c$. 
If we assume that the solutions of Eq. (3) take the form

$$
y_{k}=Y_{k} e^{j\left(\Omega_{c} t-n \theta\right)},
$$

where $n$ is the revolution harmonic, $\theta$ is the angle along the closed orbit of the accelerator, $Y_{k}$ is the amplitude of the motion for the $k$ th particle, and $\Omega_{c}$ is the collective oscillation angular frequency of the particles, then when we substitute it into Eq. (2), it becomes

$$
\left[\left(Q \omega_{0}\right)^{2}-\left(\Omega_{c}-n \omega_{0}\right)^{2}\right] Y_{k}=-j \frac{q I Z_{\perp}}{2 \pi R}\langle y\rangle .
$$

Now when we divide Eq. (5) with the term on the righthand side and then integrate both sides over the transverse beam distribution $\rho(\delta)$, the left-hand side becomes $\int \rho(\delta) y_{k} d \delta=\langle y\rangle$. Here, we have defined $\delta \equiv \Delta p / p$. The result is the dispersion relation

$$
1=j \frac{\beta q I Z_{\perp}}{2 \pi R \gamma m} \int d \delta \frac{\rho(\delta)}{\left(\Omega_{c}-n \omega_{0}\right)^{2}-\left(Q \omega_{0}\right)^{2}},
$$

where $\gamma m c^{2}$ is the total energy of each particle.

If we expand the $Q$ and $\omega_{0}$ in terms of $\delta$, we obtain

$$
Q(\delta)=Q+\xi \delta+\frac{\xi^{\prime}}{2} \delta^{2} \quad \omega(\delta)=\omega_{0}-\eta \omega_{0} \delta .
$$

Here $\xi=d Q / d \delta$ is called the linear chromaticity, $\xi^{\prime}$ the second order chromaticity, and $\eta$ is the phase slip parameter. Linearizing the denominator and keeping only the first order term in $\delta$ we obtain

$$
\begin{aligned}
1= & j \frac{\beta q I Z_{\perp}}{4 \pi R \gamma m Q \omega_{0}} \\
& \times \int d \delta \frac{\rho(\delta)}{\Omega_{c}-(n+Q) \omega_{0}-[\xi-(n+Q) \eta] \omega_{0} \delta} .
\end{aligned}
$$

It is customary to define new variables $V$ and $U$ which are proportional to the real and imaginary parts of the impedance,

$$
V+j U=\frac{q \beta I Z_{\perp}}{4 \pi R \gamma m Q \omega_{0}} .
$$

Now we can write the dispersion relation in a more compact form,

$$
(-U+j V)^{-1}=\int \frac{\rho(\delta)}{\Omega_{c}-\omega_{n}(\delta)} d \delta,
$$

where we have defined

$$
\omega_{n}(\delta)=(n+Q) \omega_{0}+[\xi-(n+Q) \eta] \omega_{0} \delta .
$$

If we consider a Gaussian distribution,

$$
\rho(\delta)=\frac{1}{\sqrt{2 \pi} \sigma_{\delta}} e^{-\left(\delta-\delta_{0}\right)^{2} /\left(2 \sigma_{\delta}^{2}\right)},
$$

where $\delta_{0}$ is the collective mean relative momentum, then Eq. (10) can be transformed into

$$
(v+j u)^{-1}=\frac{j}{\pi} \int_{-\infty}^{\infty} \frac{e^{-t^{2}}}{Z-t} d t
$$

where

$u=\frac{\sqrt{\pi} U}{\sigma_{\omega}} \quad v=\frac{\sqrt{\pi} V}{\sigma_{\omega}} \quad Z=\frac{\Omega_{c}-(n+Q) \omega_{0}}{\sigma_{\omega}}-\frac{\delta_{0}}{\sqrt{2} \sigma_{\delta}}$

and

$$
\sigma_{\omega}=\sqrt{2}[\xi-(n+Q) \eta] \omega_{0} \sigma_{\delta}
$$

Equation (13) has a known solution in the form of the complex error function $\operatorname{erfc}(z)=1-\operatorname{erf}(z)$,

$$
(v+j u)^{-1}=e^{-Z^{2}} \operatorname{erfc}(-j Z) .
$$

We can calculate $u$ and $v$ for the Tevatron with Eq. (16) from its measured parameters. The impedance of the Tevatron at $150 \mathrm{GeV}$ has been studied extensively [4,5] and the effective transverse impedance has been estimated to be $Z_{1, \perp}^{\text {eff }} \approx(1-j) \mathrm{M} \Omega / \mathrm{m}$ which for one coalesced proton bunch with intensity $N_{p}=2.6 \times 10^{11}$ particles yields a coherent tune shift $\delta Q_{\text {coh }}=-(1.0+1.0 j) \times$ $10^{-4}$. From these values, $u$ and $v$ can be estimated to be 0.22 and 0.027 , respectively, for $n=0, \xi=4.7, \eta=$ $2.8 \times 10^{-3}, \sigma_{\delta}=4.93 \times 10^{-4}$. If we assume that the beam is matched to the bucket, we can calculate $\sigma_{\omega}$ from $\sigma_{\delta}$ and we get $\sigma_{\omega}=969.7 \mathrm{~s}^{-1}$. This yields a growth rate of $\operatorname{Im}\left(\Omega_{c}\right)=-0.031 \sigma_{\omega} \mathrm{s}^{-1}$.

In Fig. 1, we plot $u$ versus $v$ for three different $\delta_{0}$ offsets which are typically used for chromaticity measurements in the Tevatron,

If we now consider the effects of second order chromaticity, we can expand Eq. (11) to second order in $\delta$ to get

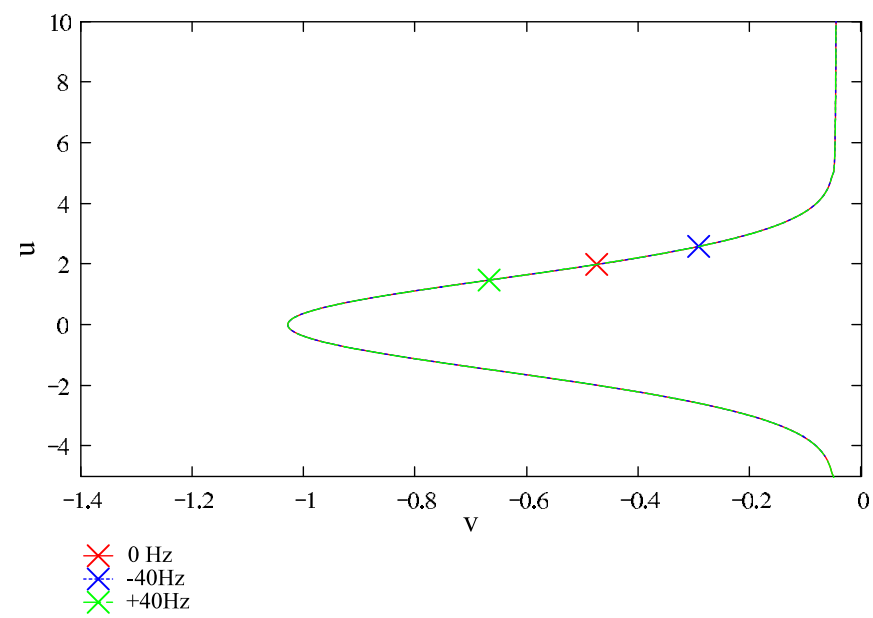

FIG. 1. The normalized $u$ versus $v$ curves for a Gaussian distribution is plotted with the growth rate $\operatorname{Im}\left(\Omega_{c}\right)=$ $-0.031 \sigma_{\omega}$ for three $\delta_{0}$ offsets $(40,0,-40) \mathrm{Hz}$. All three curves lie on top of each other. The $\times$ symbols mark the position of the coherent tune shift for each $\delta_{0}$. 


$$
\begin{aligned}
\omega_{n, \omega}(\delta)= & (n+Q) \omega_{0}+[\xi-(n+Q) \eta] \omega_{0} \delta \\
& +\left(\frac{\xi^{\prime}}{2}-\xi \eta\right) \omega_{0} \delta^{2} .
\end{aligned}
$$

Now Eq. (13) becomes

$$
\begin{aligned}
(v+j u)^{-1} & \\
& =\frac{j}{\pi} \int_{-\infty}^{\infty} d t \frac{e^{-t^{2}}}{\left(Z-\frac{\delta_{0}^{2} a_{w}}{\sigma_{\omega}}\right)-\left(1+\frac{a_{w} 2 \sqrt{2} \sigma_{\delta} \delta_{0}}{\sigma_{\omega}}\right) t-\left(\frac{a_{w} 2 \sigma_{\delta}^{2}}{\sigma_{\omega}}\right) t^{2}}
\end{aligned}
$$

where we have defined

$$
a_{w}=\left(\frac{1}{2} \xi^{\prime}-\xi \eta\right) \omega_{0} .
$$

The denominator in Eq. (17) can be factored and recast as

$$
\begin{aligned}
(v+j u)^{-1} & =-\frac{j}{\pi d} \int_{-\infty}^{\infty} d t \frac{e^{-t^{2}}}{(t+b+g)(t+b-g)} \\
d & =\frac{2 \sigma_{\delta}^{2} a_{\omega}}{\sigma_{\omega}} \\
b & =\frac{1+\frac{a_{\omega} 2 \sqrt{2} \sigma_{\delta} \delta_{0}}{\sigma_{\omega}}}{2 d} \\
c & =\frac{-Z+\frac{\delta_{0}^{2} a_{\omega}}{\sigma_{\omega}}}{d} \\
g & =\sqrt{b^{2}-c}
\end{aligned}
$$

Using MATHEMATICA [6] this integral can be solved, yielding

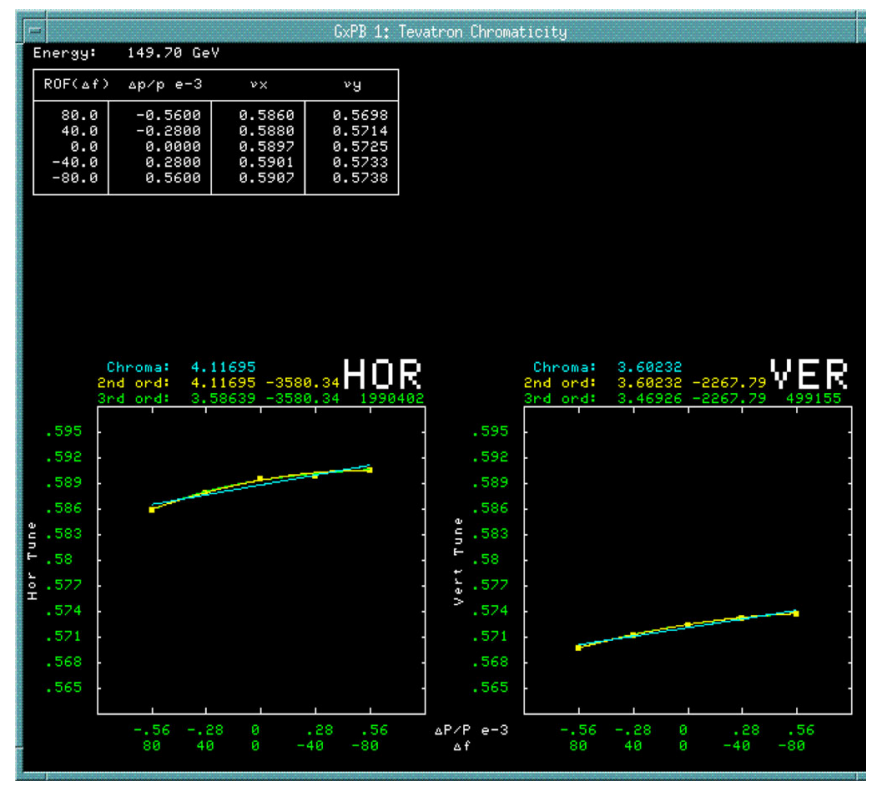

FIG. 2. The normalized $u$ versus $v$ curves for a Gaussian distribution are plotted with growth rate $\operatorname{Im}\left(\Omega_{c}\right)=-0.031 \sigma_{\omega}$ for three $\delta_{0}$ offsets $(40,0,-40) \mathrm{Hz}$ in the presence of second order chromaticity set to -4000 units. Unlike Fig. 1, the curves are now separated for each $\delta_{0}$. The $\times$ symbols mark the position of the coherent tune shifts for each $\delta_{0}$.

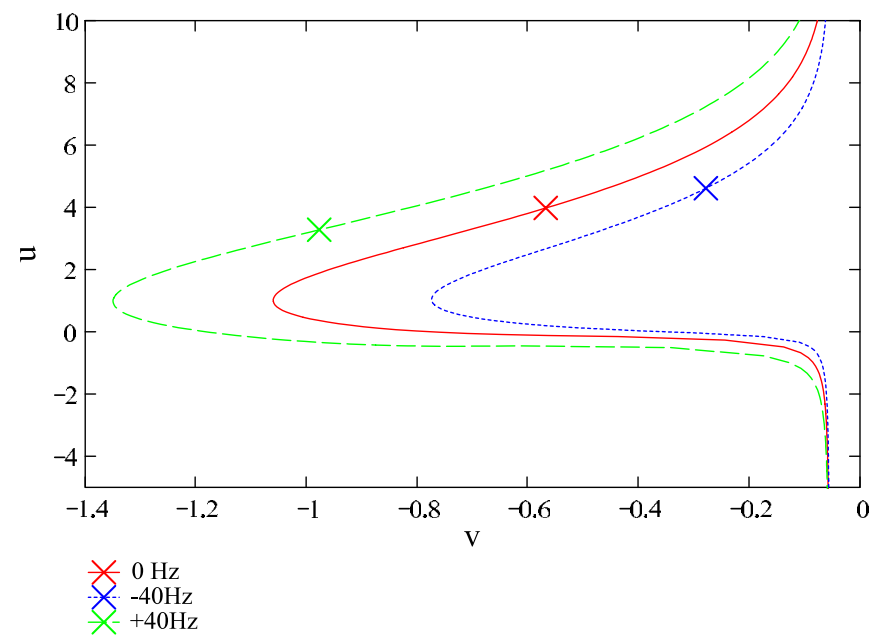

FIG. 3. Snapshot of a typical second order chromaticity measurement at injection energy in the Tevatron.

$$
\begin{aligned}
(v+j u)^{-1}= & -\frac{j}{2 d \pi g} e^{-(g+b)^{2}}\{-\pi \operatorname{erfi}(g+b) \\
& -e^{4 g b}[\pi \operatorname{erfi}(g-b)-\ln (g-b) \\
& -\ln (-g+b)]-\ln (-g+b)-\ln (g+b)\} .
\end{aligned}
$$

When we plot $u$ versus $v$ for the same $\operatorname{Im}\left(\Omega_{c}\right)=-0.031 \sigma_{\omega}$ in Fig. 2, we can see a clear $\delta_{0}$ dependence in the curves. Typically second order chromaticity at injection in the Tevatron has been found to be between \pm 1000 to \pm 5000 units in both planes (see Fig. 3). The second order chromaticity has introduced an additional $\delta_{0}$ dependence apart from the normal first and second order chromaticity effects.

This shift will impact the measured chromaticity when the traditional method is used. This effect has been postulated in our previous paper [7] and is due to the mixing of the wakefield and the higher order chromaticity.

\section{EXPERIMENTAL RESULTS}

Ever since 2005, measurements from several experiments have shown a consistent difference between chromaticity measurements performed on uncoalesced and coalesced protons at the Tevatron injection energy. These measurements are shown in Fig. 4 and in Table I. There is a consistent though varied depression in the chromaticity measured as one goes from uncoalesced to coalesced protons.

\section{A. BTF experiments}

This experience prompted us to perform more careful beam transfer function (BTF) measurements on coalesced and uncoalesced protons in the Tevatron on the central orbit at $150 \mathrm{GeV}$. The experimental setup is shown in Fig. 5 where a vector signal analyzer (VSA) is used to 


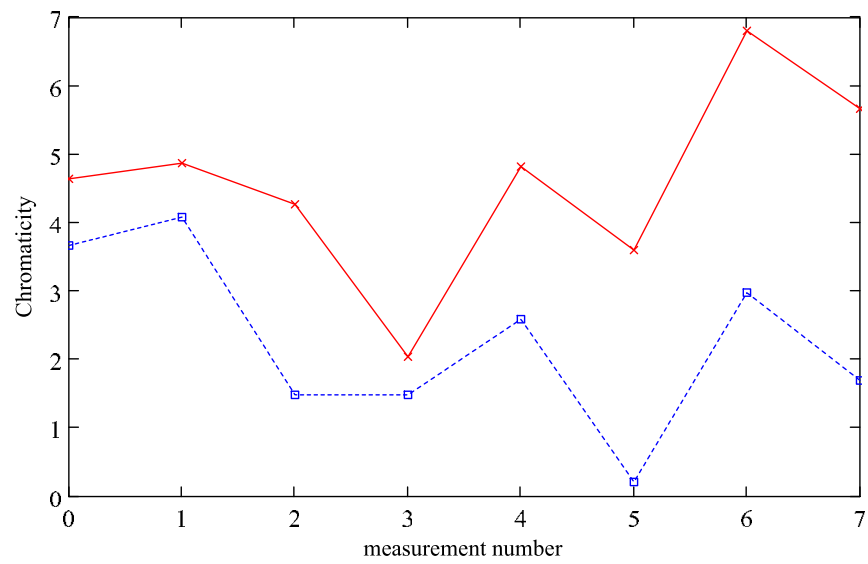

FIG. 4. Measurements made on several occasions since 2005, showing a consistent difference between coalesced and uncoalesced proton chromaticity measurements.

measure the BTF. The excitation, which is a chirp centered at the nominal betatron tune and a span from 0.568 to 0.578 tune units, from the VSA is up-converted by $(21.4+\delta f) \mathrm{MHz}$ and sent to a stripline kicker [8]. The kicker excites the beam transversely and its transverse motion is detected with a resonant Schottky detector. This signal is down-converted and measured by the VSA at baseband where it calculates the BTF from this signal and the chirp excitation.

The BTF for the two rf frequency changes $\pm 40 \mathrm{~Hz}$ and the nominal $\mathrm{rf}$ frequency at zero frequency change are measured for both uncoalesced and coalesced beam. The phase of the BTF is recorded and used for the analysis because of the way linear chromaticity is measured with a phase locked loop (PLL) based chromaticity tracking system where only the "zero" crossings of the phase response matter [9]. Figures 6 and 7 show the typical phase of the BTF measurements for three different $\delta_{0}$ changes. The $X$ symbols mark the zero crossings where the PLL can lock to thee phase offset in the PLL electronics is chosen so that it locks to a point which is symmetric about the central dip. We define this point to be the betatron tune.

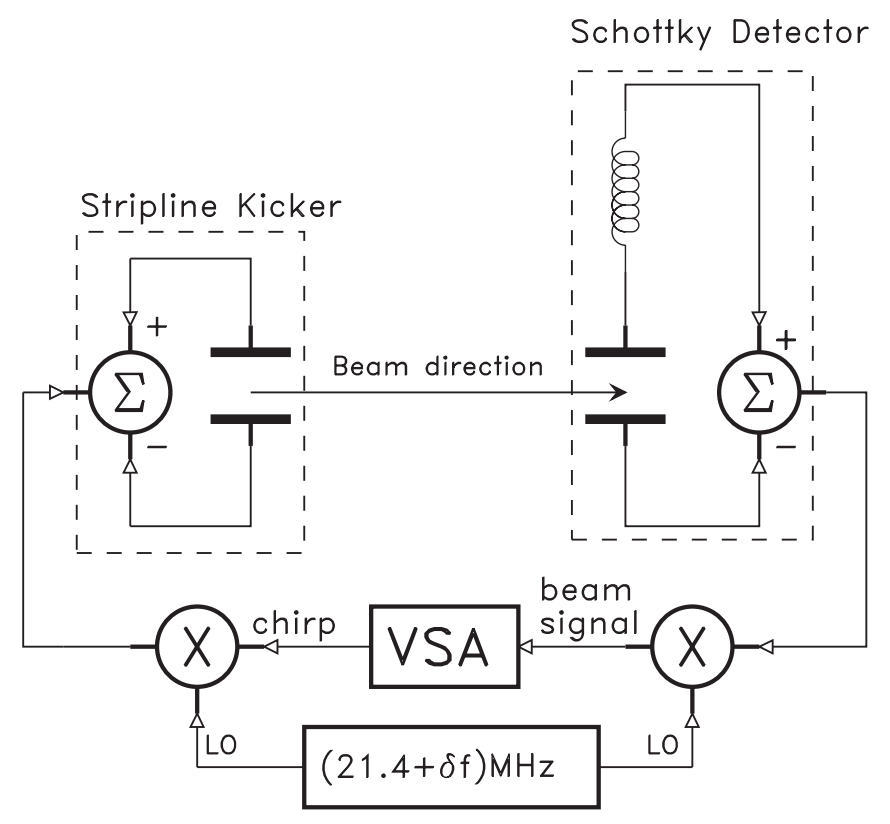

FIG. 5. A simplified block diagram of the setup used for measuring the BTF of the beam. The beam is excited with a stripline kicker where the excitation source is an up-converted chirp signal from the vector signal analyzer (VSA). The downconverted Schottky signal of the beam and the chirp excitation are used by the VSA to calculate and display the BTF.

The other dips to the left and right of the $\times$ symbols correspond to the other synchrotron resonances.

\section{B. Difference in tune shift between coalesced and uncoalesced protons}

Careful analysis of the BTF phase response shows that the tune measurements are different between uncoalesced and coalesced beam because of its dependence on $\delta_{0}$. This results in a difference in the measured chromaticity between these two bunch structures where we find that the chromaticity of the coalesced beam is always smaller than for uncoalesced beam. Figures 6 and 7 show where we have set the zero crossings and thus the measured betatron tunes. These numbers are tabulated in Tables II and III.

TABLE I. Table of measured chromaticities for uncoalesced and coalesced protons. The last column indicates number of points used to calculate linear and 2nd order chromaticity values.

\begin{tabular}{lcccc}
\hline \hline $\begin{array}{l}\text { Coalesced } \\
\text { intensity } 10^{11}\end{array}$ & $\begin{array}{c}\text { 2nd order } \\
\text { chromaticity }\end{array}$ & $\begin{array}{c}\text { Uncoalesced } \\
\text { chromaticity }\end{array}$ & $\begin{array}{c}\text { Coalesced } \\
\text { chromaticity }\end{array}$ & $\begin{array}{c}\text { Number of } \\
\text { measurement points }\end{array}$ \\
\hline 2.9 & -3447 & 4.7 & 3.7 & 3 \\
2.9 & +1043 & 4.9 & 4.1 & 3 \\
2.9 & -4146 & 4.3 & 1.5 & 3 \\
2.9 & -1382 & 2.0 & 1.5 & 3 \\
2.5 & +5454 & 4.8 & 2.6 & 14 \\
2.5 & -11940 & 3.6 & 0.2 & 14 \\
1.9 & -4033 & 6.8 & 3.0 & 14 \\
1.9 & -5910 & 5.7 & 17 & 14 \\
\hline \hline
\end{tabular}




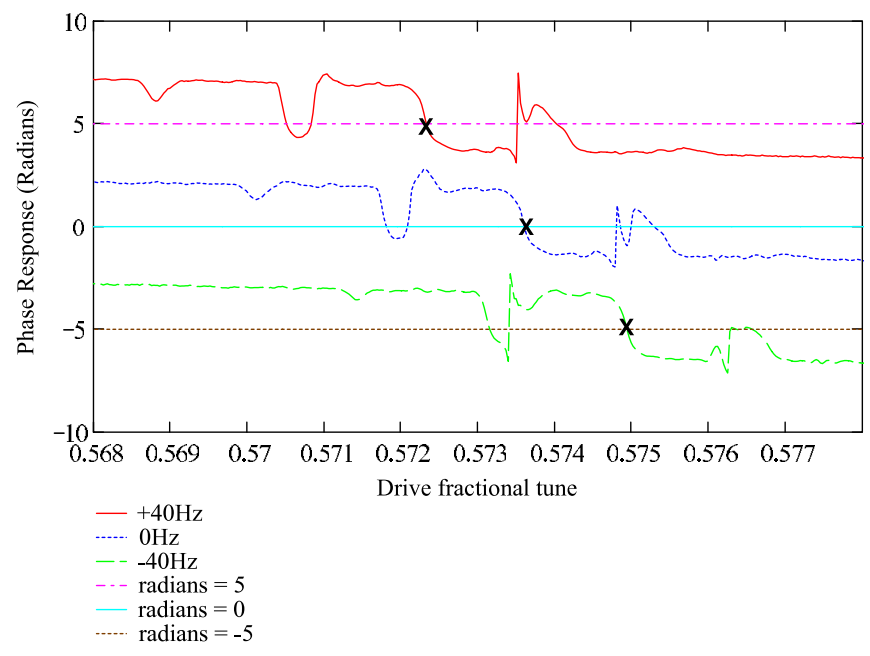

FIG. 6. The phase of the BTF for uncoalesced protons at different frequency offsets (i.e. different $\delta_{0}$ ). The $\times$ symbols mark the zero crossing for the phase which we have defined as the betatron tune.

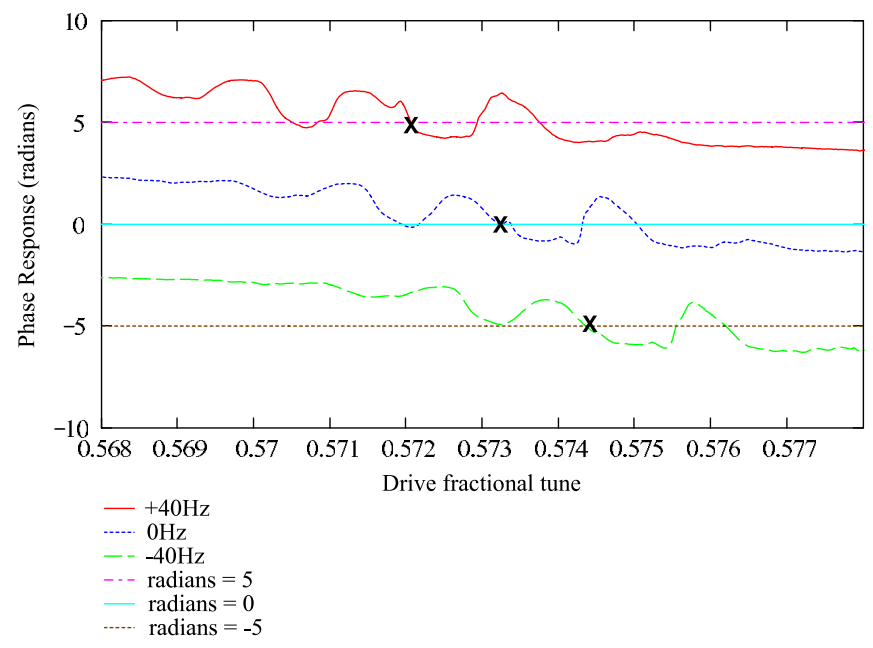

FIG. 7. The phase of the BTF for coalesced protons at different frequency offsets (i.e. different $\delta_{0}$ ) instead of uncoalesced protons which was shown in Fig. 6.

In the analysis of the data, we calculate the zero crossings using linear interpolation and root finding routines.

For example, when we consider measurements taken with sextupole magnet setting CYINJ $=27$ (see Table II), we obtain the measured phase response shown in Fig. 6 for uncoalesced protons and Fig. 7 for coalesced protons. In both cases, when the $\mathrm{rf}$ frequency is shifted by $\Delta f= \pm 40 \mathrm{~Hz}$, we expect $\delta_{0}= \pm 2.69 \times 10^{-4}$ for $f_{\mathrm{rf}}=53.104 \mathrm{MHz}$ and $\eta=0.0028$ by using the formula $\Delta f / f_{\text {rf }}=\eta \delta_{0}$.

Let us look at the data in Table II. The tune difference between uncoalesced and coalesced beam $(\Delta)$ for $\delta_{0}=0$ is $-2.0 \times 10^{-4}$. This difference is close to what we expect from the coherent tune shift caused by the resistive wall impedance $[4,5]$. However, when we compare $\Delta$ for $\delta_{0}=$ $\pm 2.69 \times 10^{-4}$, we see that the difference is large enough
TABLE II. Table of tunes and fitted chromaticities from the BTF measurements for uncoalesced and coalesced protons for chromaticity sextupole setting CYINJ $=27$.

\begin{tabular}{|c|c|c|c|c|}
\hline$\delta_{0} 10^{-4}$ & $\begin{array}{l}\text { Uncoalesced } \\
\text { tune }\end{array}$ & $\begin{array}{l}\text { Coalesced } \\
\text { tune }\end{array}$ & $\begin{array}{c}\Delta 10^{-4} \\
\text { tune }\end{array}$ & $\begin{array}{c}\Delta-\Delta\left(\delta_{0}=0\right) \\
10^{-4}\end{array}$ \\
\hline-2.69 & 0.57233 & 0.57206 & -2.7 & -0.7 \\
\hline 0.0 & 0.57360 & 0.57340 & -2.0 & 0.0 \\
\hline 2.69 & 0.57494 & 0.57435 & -5.9 & -3.9 \\
\hline Chromaticity & 4.85 & 4.26 & -0.59 & \\
\hline
\end{tabular}

TABLE III. Table of tunes and fitted chromaticities from the BTF measurements for uncoalesced and coalesced protons for chromaticity sextupole setting CYINJ $=33$.

\begin{tabular}{|c|c|c|c|c|}
\hline$\delta_{0} 10^{-4}$ & $\begin{array}{c}\text { Uncoalesced } \\
\text { tune }\end{array}$ & $\begin{array}{l}\text { Coalesced } \\
\text { tune }\end{array}$ & $\begin{array}{c}\Delta \text { tune } \\
10^{-4}\end{array}$ & $\begin{array}{c}\Delta-\Delta\left(\delta_{0}=0\right) \\
10^{-4}\end{array}$ \\
\hline-2.69 & 0.57230 & 0.57226 & -0.4 & 3.3 \\
\hline 0.0 & 0.57369 & 0.57332 & -3.7 & 0.0 \\
\hline 2.69 & 0.57480 & 0.57430 & -5.0 & -1.3 \\
\hline Chromaticity & 4.65 & 3.78 & -0.87 & \\
\hline
\end{tabular}

to alter the measured linear chromaticity with the traditional method. This is the reason why the linear chromaticity for coalesced beam can be underestimated because the measurements of the zero phase crossings have been shifted from where we expect them to be. In this case we see that the measured chromaticity of the coalesced beam smaller than the coalesced beam by 0.59 units.

We repeated the experiment for chromaticity sextupole setting CXINJ = 33 units and we can see from Table III that $\Delta$ shows the same type of tune differences between uncoalesced and coalesced beam for different $\delta_{0}$ values. Again, the measured linear chromaticity for coalesced beam is smaller than uncoalesced beam by 0.87 units.

The data clearly shows that $\Delta$ has a $\delta_{0}$ dependence. Naively we might expect that the effect of impedance will shift the coherent tune uniformly with little or no dependence on $\delta_{0}$, i.e., the coherent betatron tune shift is effectively decoupled from $\delta_{0}$. We can explain this observation with our calculation in Sec. II where we have

TABLE IV. Table of tunes and fitted chromaticities using Eq. (21) for uncoalesced and coalesced protons, assuming $\Delta=-2.25 \times 10^{-4}, \xi=4.7$, and $\xi^{\prime}=-4000$.

\begin{tabular}{lcccc}
\hline \hline & \multirow{2}{*}{$\begin{array}{c}\text { Uncoalesced } \\
\delta_{0} 10^{-4}\end{array}$} & tune & Coalesced & \multicolumn{2}{c}{$\Delta$} & $\Delta-\Delta\left(\delta_{0}=0\right)$ \\
\hline-2.69 & 0.57232 & 0.57199 & -3.3 & -1.1 \\
0.0 & 0.57358 & 0.57336 & -2.2 & 0.0 \\
2.69 & 0.57484 & 0.57423 & -6.1 & -3.9 \\
Chromaticity & 4.70 & 4.16 & -0.54 & \\
\hline \hline
\end{tabular}


shown that $\delta_{0}$ is coupled to the coherent tune shift when second order chromaticity is included. We can use Eq. (21) to generate Table IV to compare the theory to the experimental data. The results show that the predictions of our simple model match the characteristics of the experiment surprisingly well. The theory shows that there is an asymmetry in $\Delta$-larger shift for positive $\delta_{0}$ shift than negative - and the size of $\Delta$ for the different $\delta_{0}$ 's are comparable with the experimental data shown in Tables II and III.

\section{FULL 6D SIMULATION USING BBSIM CODE}

To further verify the effects of higher order chromaticity and wakefields on the measured linear chromaticity, we added the ability to handle single bunch resistive wall wakefields and second order chromaticity to BBSIMC. BBSIMC was originally conceived as a parallel weak strong beam-beam simulation code developed by Sen at FNAL [10].

The simulation was set up to model the BTF measurement as closely as possible. The whole simulation consisted of ten frequency sweeps of the kicker back and forth across the resonant betatron tune. Each sweep totalled about $19 \times 10^{3}$ turns. The average beam position at the pickup was then recorded for each turn. The simulation was further enabled to model an offset in energy due to a change in frequency that is done during a chromaticity measurement. The resistive wall wakefield was applied using a simple $1 / \sqrt{r}$ model with the effects lumped into a single location in the ring. Linear and higher order chromaticity was modeled using kicks distributed around the ring. Our simulations typically ran between $3 \times 10^{4}$ to $3 \times 10^{5}$ particles.

Although part of the experiment was done using four coalesced bunches to improve the signal to noise ratio of the measurement, the primary transverse wakefield effect from the resistive wall goes as $z^{-1 / 2}$ and since the rms bunch size is $78 \mathrm{~cm}$ and the interbunch spacing is 21 buckets or $118 \mathrm{~m}$, the interbunch effects are about a factor of 12 smaller than the intrabunch effects. For this reason we will neglect the long range wakefield effects. We will benchmark BBSIMC against the BTF measurements to see whether we can reproduce it.

For uncoalesced protons we are able to reproduce fairly accurately the phase of the BTF. Figure 8 shows the BBSIMC results overlaid onto the measured BTFs.

For coalesced protons, when we include resistive wall wakefields in the BBSIMC model together with second order chromaticity, we find that while the phase of the simulated BTFs begins to deviate from the uncoalesced simulations it is not an exact match to the measured BTFs. This is in part due to the fact that our model has only simulated chromatic effects to second order, and recent evidence [11] suggests that the distribution in $\delta$ due to the coalescing process yields a persistent longitudinal dipole mode which distorts the

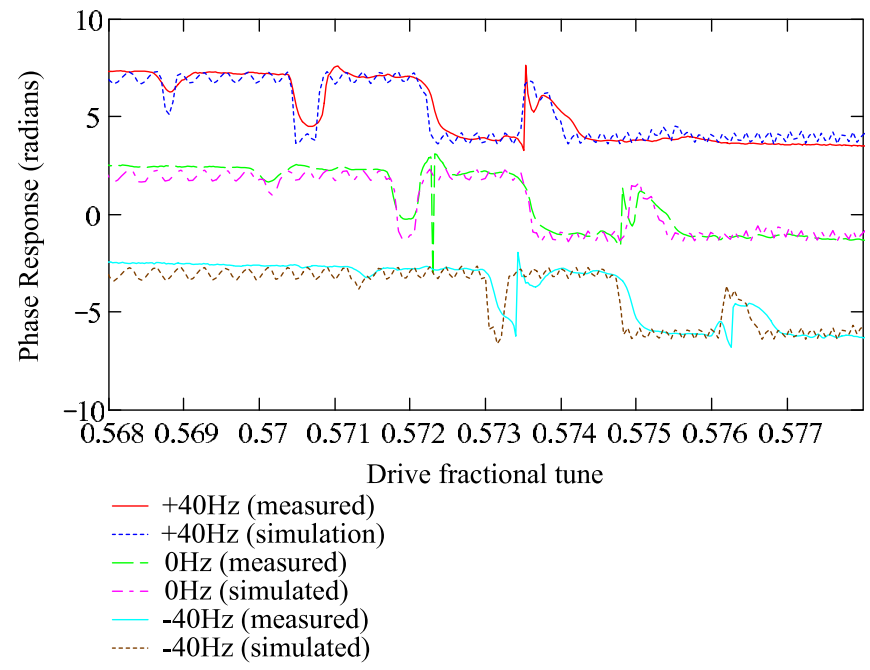

FIG. 8. The phase of the BTF for uncoalesced protons at different frequency offsets (i.e. different $\delta_{0}$ ). Overlaid are the simulated results using BBSIMC with 4.7 units of linear chromaticity, $-2 \times 10^{3}$ units of second order chromaticity.

longitudinal distribution unlike the smooth Gaussian distribution that we have assumed. Figure 9 shows the BBSIMC results overlaid on the measured BTFs.

From Fig. 10 we can obtain a better sense of the magnitude of this effect and its response to intensity and positive and negative second order chromaticity values. We see that generally, as was observed in experiment, that coalesced measurements produce lower linear chromaticity values than uncoalesced. However, the correlations between linear chromaticity and intensity and second order chromaticity appears nontrivial (not simply linear).

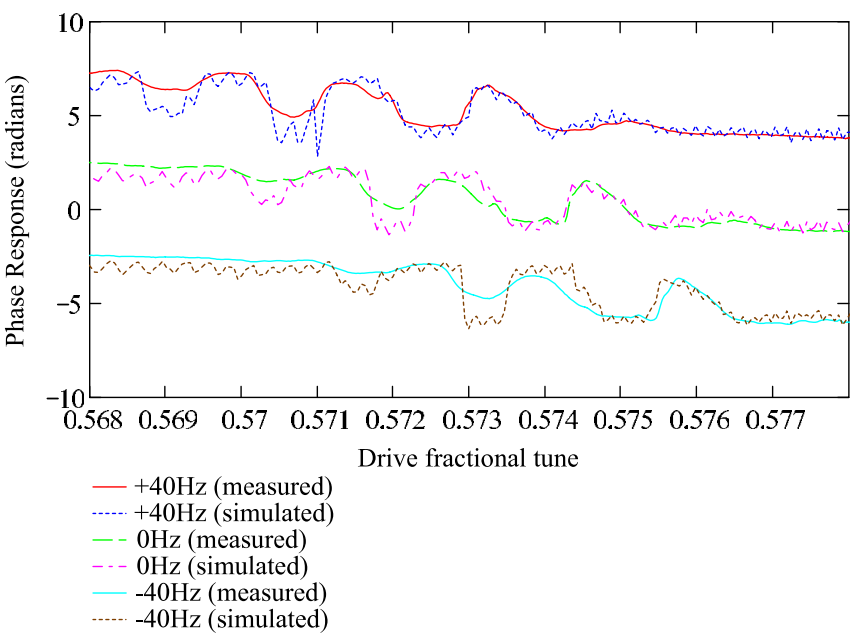

FIG. 9. The phase of the BTF for coalesced protons at different frequency offsets (i.e. different $\delta_{0}$ ). Overlaid are the simulated results using BBSIM with 4.7 units of linear chromaticity, $-2 \times 10^{3}$ units of second order chromaticity, resistive wall wakefield, and the intensity of $3.0 \times 10^{11}$ protons. 

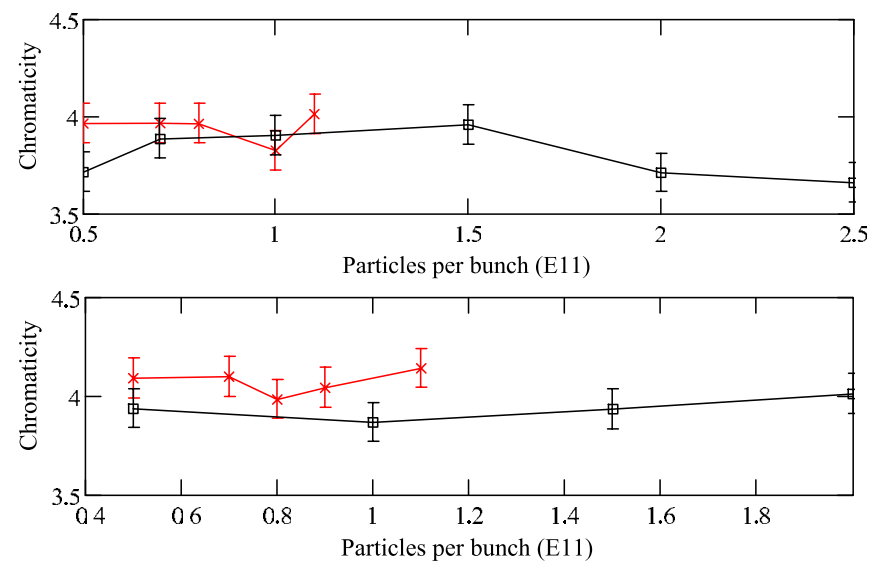

FIG. 10. Plot of the results of the $6 \mathrm{D}$ simulations with $3 \times 10^{5}$ particles using BBSIM. The simulation was set up to imitate a chromaticity measurement using the BTF method. We used 4.0 units of linear chromaticity. The top plot shows simulations with $-4 \times 10^{3}$ units of second order chromaticity, the bottom plot with $+4 \times 10^{3}$ units of second order chromaticity. In these plots the red trace $(X)$ shows uncoalesced protons, the black trace $(\square)$ shows coalesced protons. These are all plotted with intensities up to instability threshold with only transverse resistive wall impedance effects (no longitudinal).

\section{CONCLUSION}

We have shown that the naive expectation that the coherent betatron tune shifts from $\delta_{0}$ changes are independent of bunch structure is false. In fact, our experiments show that the amount of coherent tune shift is strongly dependent on bunch structure and therefore wakefields. When we include higher order chromaticity, we find that the net coherent tune shift has a significant dependence on $\Delta p / p$. We have demonstrated this by using simple analytical models and full 6D simulations which explain the experimental observations.

\section{ACKNOWLEDGMENTS}

This work was operated by Fermi Research Alliance, LLC under Contract No. DE-AC02-07CH11359 with the
United States Department of Energy, was also supported by SBIR-DOE-NP Grant No. DE-FG02-08ER85183, and had partial support from Tech-X Corp. This research used resources of the National Energy Research Scientific Computing Center, which is supported by the Office of Science of the U.S. Department of Energy under Contract No. DE-AC02-05CH11231.

[1] Uncoalesced beam in the Tevatron means that there are 30 bunches in adjacent buckets.

[2] One coalesced bunch means that seven bunches from the main injector are coalesced into one high current bunch and injected into the Tevatron. For our experiment, four coalesced bunches separated by 21 buckets are used.

[3] S. Y. Lee, Accelerator Physics (World Scientific, Singapore, 1999), pp. 199-206.

[4] P. M. Ivanov et al., in Proceedings of the 20th Particle Accelerator Conference, Portland, OR, 2003 (IEEE, New York, 2003), p. 3062; in Proceedings of the 21st Particle Accelerator Conference, Knoxville, 2005 (IEEE, Piscataway, NJ, 2005), p. 1714; in Proceedings of the 21st Particle Accelerator Conference, Knoxville, 2005 (IEEE, Piscataway, NJ, 2005), p. 2756; P. M. Ivanov, F0 Lambertson liner review (2003), internal FNAL document.

[5] V.H. Ranjbar and P. Ivanov, Phys. Rev. ST Accel. Beams 11, 084401 (2008).

[6] http://www.wolfram.com/.

[7] C. Y. Tan and V.H. Ranjbar, Phys. Rev. ST Accel. Beams 11, 032802 (2008).

[8] There is a small change in the local oscillator (LO) frequency of $\delta f<20 \mathrm{~Hz}$ used for up-conversion and down-conversion because the low level rf, from which the LO of $21.4 \mathrm{MHz}$ is derived, is changed for chromaticity measurements.

[9] C. Y. Tan, Nucl. Instrum. Methods Phys. Res., Sect. A 602, 352 (2009).

[10] http://www-ap.fnal.gov/ hjkim/.

[11] A. Burov and C.Y. Tan, in Proceedings of 2011 Particle Accelerator Conference, New York, NY, WEP116 [http://www.c-ad.bnl.gov/pac2011/proceedings/ papers/wep116.pdf]. 